\title{
Classification of trochlear dysplasia as predictor of clinical outcome after trochleoplasty
}

\author{
Sandro F. Fucentese $\cdot$ Patrick O. Zingg \\ Jürgen Schmitt • Christian W. A. Pfirrmann • \\ Dominik C. Meyer $\cdot$ Peter P. Koch
}

Received: 8 June 2010/Accepted: 20 January 2011/Published online: 8 February 2011

(C) Springer-Verlag 2011

\begin{abstract}
Purpose Sulcus-deepening trochleoplasty restores the trochlear groove in patients with patellofemoral instability and underlying trochlear dysplasia. There are types of dysplasia both with (B and D) and without (A and C) a supratrochlear spur. The aim of this study was to identify influencing factors for the clinical outcome following trochleoplasty.

Methods Forty-four knees in 38 patients who underwent trochleoplasty for instability (type A in 9, B in 15, C in 9 and $\mathrm{D}$ in 11 knees) were assessed clinically with the Kujala score and radiologically with radiographs and MRI. The median follow-up was 4 (2-7.8) years.

Results At follow-up, the median Kujala score had improved from $68(29-84)$ to $90 \quad(42-100)$ points $(P<0.001)$. Instability $(P<0.001)$ and pain $(P=0.027)$ decreased significantly, but in 3 knees, pain was worse postoperatively. Twenty-seven knees were ranked as excellent, 10 as good, 2 as fair and 5 as poor. Overall, dysplasia types B and D benefited more from surgery than types A and C. The postoperative MRI revealed no chondrolysis or subchondral necrosis, but deterioration of cartilage on the lateral trochlear facet was identified.

Conclusion Trochleoplasty is a useful and reliable surgical technique to improve patellofemoral instability in patients with a dysplastic trochlea. While improved
\end{abstract}

S. F. Fucentese $(\bowtie) \cdot$ P. O. Zingg · J. Schmitt .

D. C. Meyer - P. P. Koch

Department of Orthopaedic Surgery, University Hospital

Balgrist, Zurich, Forchstr. 340, 8008 Zurich, Switzerland

e-mail: sandro.fucentese@balgrist.ch

C. W. A. Pfirrmann

Department of Radiology, University Hospital Balgrist, Zurich,

Forchstr. 340, 8008 Zurich, Switzerland stability is predictable, pain is less predictable and may even increase following surgery. The overall results were directly dependent on the type of the dysplasia, with a significantly better clinical outcome in type B and D. The clinical relevance of this study is that severe dysplasia can successfully be treated with trochleoplasty.

Level of evidence III.

Keywords Trochleoplasty - Patellofemoral instability . Trochlear dysplasia

\section{Introduction}

Trochlear dysplasia is a known risk factor for patellar instability. Maldegue and Malghem used a strictly lateral radiograph of the knee to analyse the trochlear depth [20]. Dejour et al. defined three types of dysplasia (I-III) using a strictly lateral knee radiograph and reported that trochlear dysplasia is prevalent in $85 \%$ in patients with recurrent patellar dislocation $[13,14]$. This observed instability has also been demonstrated in a biomechanical study [2]. Dejour et al. modified the classification introducing four types (types A-D) with the additional use of axial views acquired by CT scanning [10, 11].

Different techniques have been described to correct trochlear dysplasia [1, 3, 10, 21]. Newer techniques attempt to deepen the trochlear groove to attain a more anatomical shape in order to reduce the local pressure on the patellofemoral joint [3, 10, 15, 21, 25, 27-29]. While Masse impacted the cartilage without fixation, Dejour fixed the split cartilaginous flake after $\mathrm{V}$-shaped modelling of the groove with two K-wires. Bereiter's and Gautier's technique peels off the cartilage, deepens the groove and the cartilage bony fragment is pressed into the newly formed 
groove without splitting the cartilage and fixed with two absorbable sutures (Figs. 1-3) [25, 27-29]. Even though improved stability may be achieved in most cases,
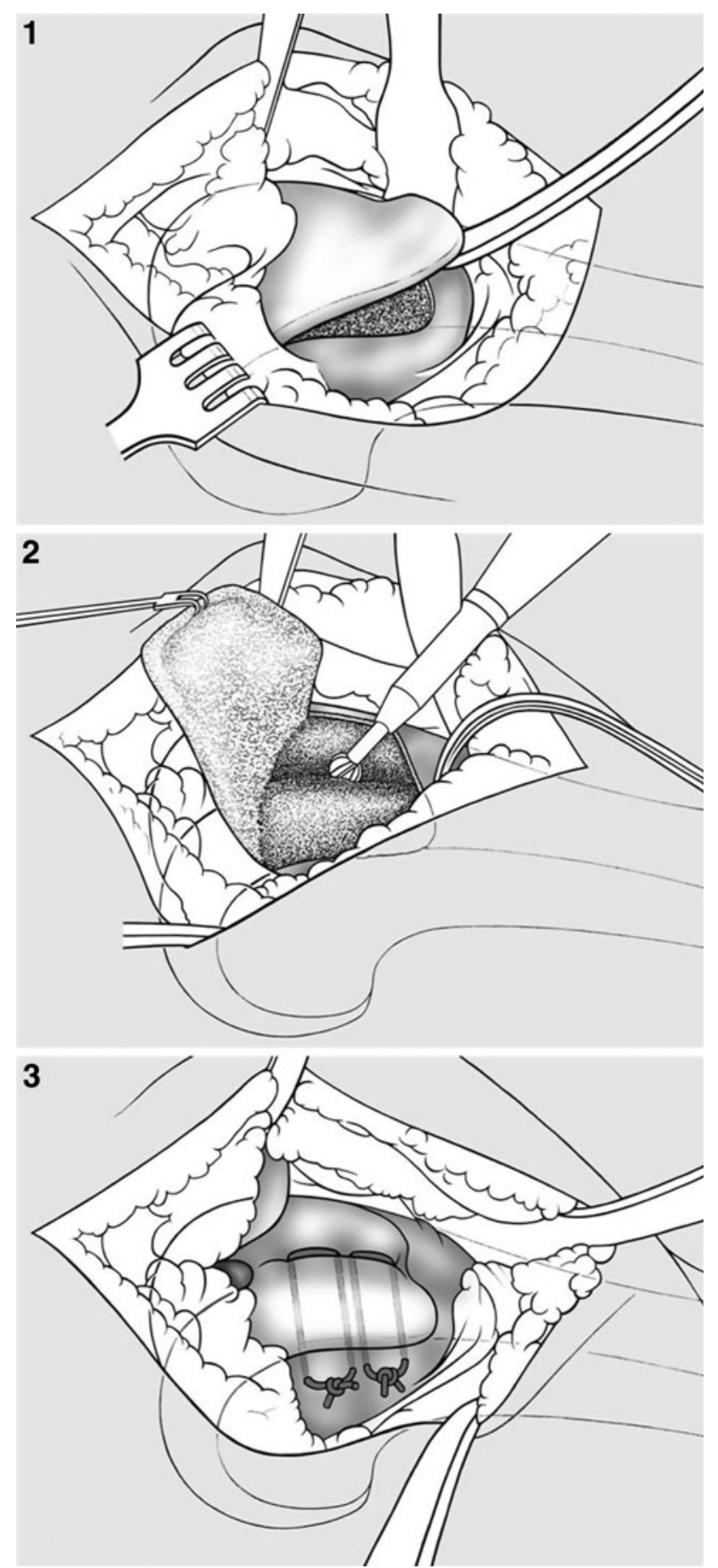

Figs. 1-3 Bereiter's and Gautier's technique for trochleoplasty: a osteochondral flake is peeled off with a chisel (1), then the groove is deepened (2), and the osteochondral fragment is pressed into the newly formed groove without splitting the cartilage and fixed with two absorbable sutures (3) unsatisfying results have been reported and have often been associated with degenerative changes [15, 25, 27-29].

The goal of this study was to examine our patients clinically (stability, pain) and radiologically (radiographs, MRI) after sulcus-deepening trochleoplasty according to Bereiter's and Gautier's technique [3] and to identify any preoperative and postoperative factors that might influence either the subjective or objective patient outcomes.

It was hypothesized that the following parameters would predict a favourable outcome: young age; minimal cartilaginous deterioration; degree of dysplasia; and isolated, mostly pain-free symptoms of instability.

\section{Materials and methods}

The study was approved by the institutional review board (IRB) of the University of Zurich (Switzerland). All patients signed an informed consent form. The data were accumulated prospectively, but analysed retrospectively. The differentiated visual analogue score (VAS) and a "subjective knee value" were assessed only at follow-up.

\section{Inclusion criteria}

Between 1999 and 2006, trochleoplasty for the treatment of trochlear dysplasia was performed in 42 patients (48 knees). The sulcus-deepening technique described by Gautier and Bereiter was utilized [3]. Indications for trochleoplasty were two or more patellar dislocations, or one patellar dislocation with a persistently positive patellar apprehension sign for more than 1 year in the presence of radiologically confirmed trochlear dysplasia [11, 13, 14]. Contraindications for trochleoplasty were radiological signs of arthritic degeneration of the patellofemoral joint or open epiphyseal plates.

All patients with a minimal follow-up of 2 years and a preoperative MRI were included. Of the original 42, 38 patients (44 knees) were available for the final follow-up. Four patients were lost to follow-up, two of whom refused further examinations for unspecified reasons and two who could not be localized.

Of the 38 patients (44 knees), there were nine with type A, 15 type $\mathrm{B}, 9$ type $\mathrm{C}$ and 11 type $\mathrm{D}$ dysplasias according to Dejour et al. [11]. Ten patients were men and 28 women. Five of the six bilateral surgeries were performed on women. The median age at surgery was $18(14-40)$ years. The median final follow-up time was at $4(2-7.8)$ years. Eleven patients (13 knees) had undergone previous knee surgery performed before the index surgery. Three knees of these 13 knees had been operated on twice, the others once. Previous surgeries included (often in combination) 
arthroscopy (10), medial reefing or plication (6), lateral release (5) and/or tuberosity transfer (3).

\section{Surgical technique}

Trochleoplasty was performed according to the technique described by Bereiter and Gautier [3, 25, 29]. Surgery is carried out under spinal anaesthesia in combination with a femoral nerve catheter. Preoperative antibiotic prophylaxis is administered. A tourniquet is applied to the thigh. After a lateral parapatellar skin incision is made, the lateral retinaculum is incised and a lateral arthrotomy is performed. The joint is inspected and the patellar shape defined. The synovium around the trochlea is dissected circumferentially. An osteochondral flake of the trochlea is peeled off in a proximal to distal direction down to the load-bearing zone of the condyles using a curved osteotome (Fig. 1). A deeper more anatomically correct trochlear groove is restored using a chisel and high-speed burr (Fig. 2). The upper part of the groove starts laterally and ends at a point established pre- and intraoperatively by considering the preoperative tibial tuberosity to trochlear groove distance (TTTG) and aiming to normalize this as much as possible. The greatest depth the groove reaches is about $6 \mathrm{~mm}$, with a smooth graduation at either end [16]. The osteochondral flake is then pressed into the newly formed groove and fixed with two transosseus vicryl no. 5 sutures (Vicryl-tape, Ethicon, Norderstedt, Germany) in the deepest part of the new trochlea (Fig. 3). The synovium is reattached to the cartilaginous edges with a continuous absorbable suture. The tendon of the vastus medialis obliquus muscle (VMO) is tightened medially and attached to the patella with one crossstitch, and the joint is closed with a continuous absorbable suture without closing the lateral retinaculum, leaving an indirect lateral release. The intraarticular drain is removed after $24 \mathrm{~h}$. Patients were mobilized with a knee brace fixed at $30^{\circ}$ flexion with full weight bearing for 6 weeks. The range of motion was allowed from 30 to $60^{\circ}$ for the first 3 weeks and from 30 to $90^{\circ}$ for the forth to sixth week.

\section{Radiological assessment}

The radiological assessment consisted of plain radiographs and MRI. The radiographs consisted of an antero-posterior view, a direct lateral and a sunrise view. Dysplasia was defined using the lateral radiograph and the most proximal MRI slice with trochlear cartilage in the axial view according to the classification of Dejour et al. [11]. Patellar height was determined using the Caton-Deschamps index on the lateral radiograph [6]. A ratio of more than 1.2 is considered a patella alta, and a ratio of less than 0.8 is a patella baja.
A standardized knee MRI protocol was used. Axial images were used to calculate the tibial tuberosity to trochlear groove distance (TTTG), patellar tilt angle, and the distance between trochlear groove and posterior patellar edge was measured $[5,8,9,16,18,24]$. The cartilage was evaluated according to Noyes et al. [22]. This classification consists of five grades. Grade 0 is a normal cartilage with a homogenous signal intensity, intact cartilage surface and thickness. Grade 1 denotes an abnormal signal intensity with focal alteration of the cartilage signal intensity, but with a normal surface. Grade 2 represents superficial fraying, erosion or ulceration with a depth of not more than $50 \%$ of cartilage thickness, whereas in grade 3 , the defect is more than $50 \%$ but less than $100 \%$. Grade 4 exhibits full-thickness cartilage damage. On both the patellar and the femoral sides, the cartilage was subdivided into three zones: medial, central and lateral (Fig. 4). The highest grade of cartilage damage was recorded for each region for evaluation. The presence of chondrolysis or necrotic cartilage was recorded.

\section{Clinical assessment}

The pre- and postoperative clinical assessment consisted of a structured interview and a standardized physical examination including walking and limping, clinical axis, Q-angle, swelling, tenderness, range of motion, muscle status, mobility of patella and patellar tilt, retropatellar compression pain and crepitus, and the apprehension test for patellar instability. This last test was performed starting in extension and finishing in $30^{\circ}$ of flexion. With this information, the Kujala score was calculated [19]. Further parameters were evaluated: aetiology of the first dislocation

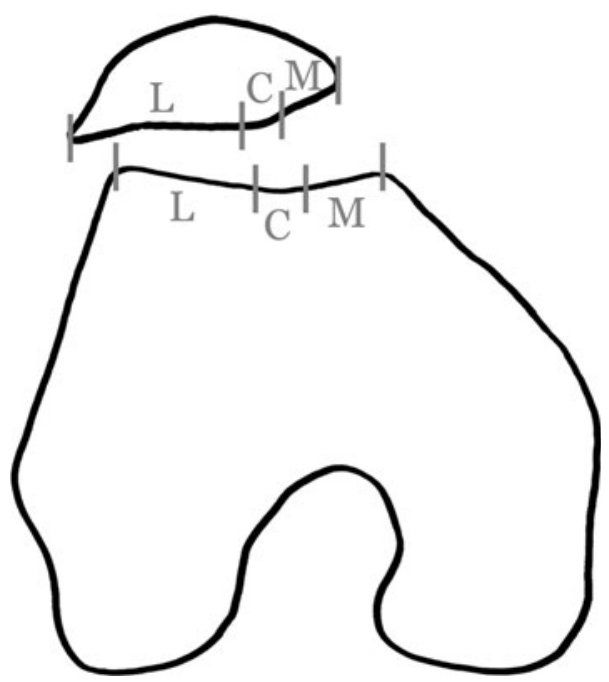

Fig. 4 On the patellar and the femoral side, the cartilage was subdivided into three zones: medial $(M)$, central $(C)$ and lateral $(L)$, for differentiated assessment of cartilaginous changes 
(atraumatic vs. traumatic), recurrent dislocations and prior surgical interventions. Postoperative pain was assessed using a differentiated visual analogue scale (VAS) for pain at rest, pain with sporting activity and the maximal pain. The scale ranged from zero (=no pain) to 100 (=very strong pain) in per cent. In the postoperative clinical assessment, the subjective symptoms (instability sensation, feeling of subluxation) and objective signs (apprehension test, documented dislocation) of stability were noted. The subjective result was ranked as excellent, good, fair or poor. Patients were asked to describe their overall satisfaction with the knee as better, unchanged or worse than preoperatively and also asked whether they would choose to have surgery again. To obtain a subjective patient's estimation for the overall knee score, the so-called "subjective knee value" was introduced at follow-up, using a score analogue to the "subjective shoulder value" [17]. Here, a healthy knee is ranked as $100 \%$, while a painful, flail knee would be ranked with $0 \%$.

\section{Statistical analysis}

Statistical analysis was performed by a professional statistical consultant. Pre- and postoperative clinical and radiological findings were compared using the Wilcoxon signed-rank test. The significance level was set at $P<0.05$. Spearman's rho test was used for the correlation.

\section{Results}

The radiological results are summarized in Table 1. See also Fig. 5a, b.

Preoperatively, ten knees had a persistent subluxation and apprehension after one dislocation, while 34 knees had recurrent patellar dislocations. All patients had a positive apprehension sign. The cause of first dislocation was significant trauma in nine knees, while in the remaining knees, there was a history of minor or inadequate trauma only. Preoperatively, four knees were painful at rest and 35 knees under load.

The median Kujala score increased from preoperatively 68 (29-84) to postoperative 90 (42-100) points $(P<0.001)$. The preoperative median value in the pain section was $8(3-10)$ points and postoperatively $8(3-10)$ points $(P=0.027)$. It was unchanged in 27 , decreased in 14 and increased in 3 knees. Three patients with ongoing pain underwent further arthroscopic debridement with the removal of loose bodies in one knee. Two of these three had a satisfactory and one an unsatisfactory result. The preoperative median value in the stability section was $0(0-6)$ and postoperative $10(0-10)$ points $(P<0.001)$. The apprehension test at follow-up remained positive in 11 knees. However, six patients reported the ongoing
Table 1 Radiological results

\begin{tabular}{llll}
\hline & $\begin{array}{l}\text { Median } \\
\text { preoperative } \\
\text { (range) }\end{array}$ & $\begin{array}{l}\text { Median } \\
\text { postoperative } \\
\text { (range) }\end{array}$ & $P$ \\
\hline $\begin{array}{l}\text { Conventional } \\
\text { radiographs }\end{array}$ & & & \\
Caton-Deschamps index & 1.04 & 0.94 & $<0.001$ \\
Patella alta/normal/baja & $11 / 29 / 4$ & $3 / 36 / 5$ & \\
MRI & & & \\
Patella medial facet & $0(0-4)$ & $0(0-4)$ & n. s. \\
Patella central & $0(0-4)$ & $2(0-4)$ & 0.002 \\
Patella lateral facet & $0(0-4)$ & $0(0-4)$ & n. s. \\
Trochlea medial & $0(0-4)$ & $1(0-4)$ & $<0.001$ \\
Trochlea central & $0(0-4)$ & $2(0-4)$ & $<0.001$ \\
Trochlea lateral & $0(0-4)$ & $1(0-4)$ & 0.006 \\
TTTG (mm) & $17(11-30)$ & $13(0-21)$ & $<0.001$ \\
Patellar tilt angle $\left({ }^{\circ}\right)$ & $32(11-60)$ & $13((-3)-36)$ & $<0.001$ \\
Translation $(m m)$ & $8((-7)-23)$ & $0((-7)-13)$ & $<0.001$ \\
Chondrolysis & No & No & \\
Subchondral necrosis & No & No & \\
\hline For & & &
\end{tabular}

For patella height, Caton-Deschamps index is used [6]. The MRI assessment shows the cartilage status according to Noyes et al. [22], the distance of tibial tubercle and trochlear groove (TTTG) according to Goutallier et al. and Schöttle et al. [18, 24]. The translation measured the distance between trochlear groove and posterior patellar edge [16]. No chondrolysis and subchondral necrosis was found

sensation of instability during the activities of daily living. There was one knee with a new recurrent atraumatic dislocation. Two patients with residual instability underwent a further stabilization surgery, comprising a reconstruction of the medial patellofemoral ligament with a satisfactory result in one knee and an anteromedialization of tibial tuberosity with a fair result in the other.

Twenty-seven knees were ranked as excellent, 10 as good, two as fair and five as poor. Five patients judged their overall situation as worse compared with preoperatively (3 type $\mathrm{A}$; each one type $\mathrm{B}$ and $\mathrm{C}$ ), two as unchanged (each one type B and C) and 31 as better.

Seven patients would not repeat surgery (4 type A or C; 3 type B). These included two patients who reported a worse situation postoperatively with persistent pain, three patients with residual instability, two with an unchanged level of sporting activity and one who felt that the rehabilitation period was too long.

Complications included one transient postoperative femoral nerve palsy after peripheral anaesthesia, one wound-healing problem and one complex regional pain syndrome. There were no infections, thromboembolic events or haematomas.

Preoperative pain correlated only with the preoperative Kujala score $(r=0.76 ; P<0.001)$. The preoperative 
Fig. 5 a Preoperative axial MR slice showing the severe dysplasia. b Postoperative axial MR slice showing the corrected trochlear groove

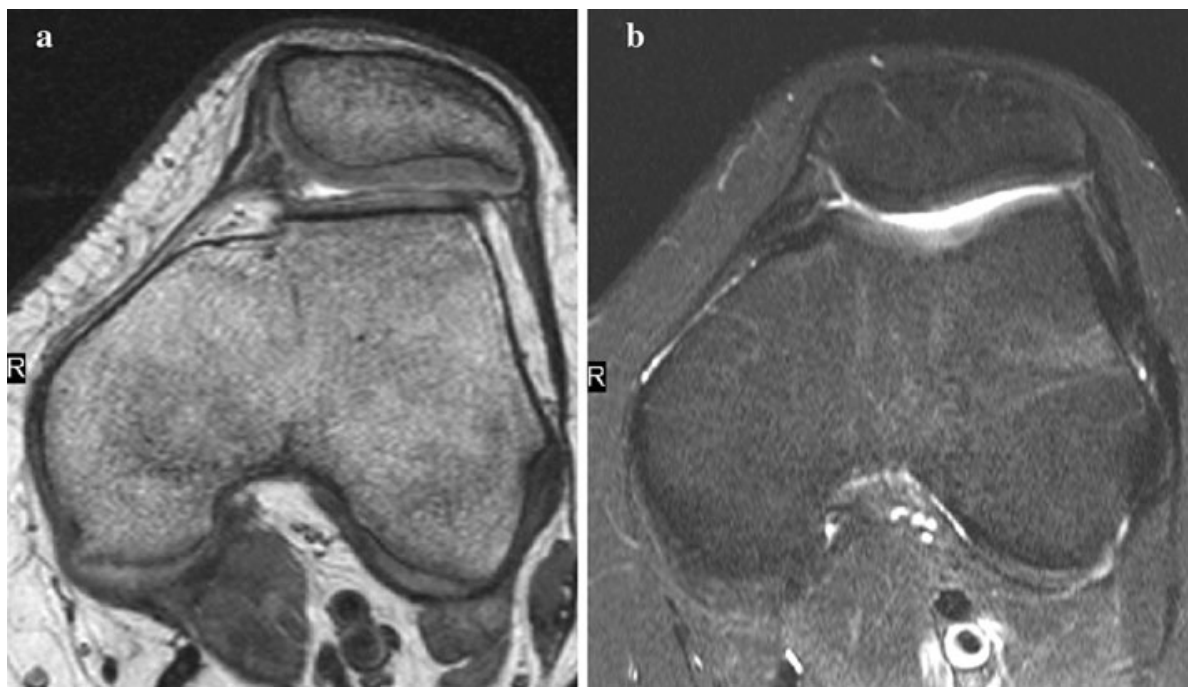

Kujala score had no correlation with the postoperative score and imaging. Postoperative pain correlated with the postoperative Kujala score $(r=0.814 ; P<0.001)$, postoperative subjective knee value $(r=0.729 ; P<0.001)$, the postoperative satisfaction $(r=0.76 ; P<0.001)$ and the VAS maximal $(r=0.741 ; P<0.001)$. There was no correlation between the postoperative pain and the preoperative clinical or radiological findings.

The subjective knee value correlated with the postoperative Kujala score $(r=0.81 ; P<0.001)$. Patients' satisfaction was positively correlated with postoperative Kujala score $(r=0.60 ; P<0.001)$, postoperative pain $(r=0.58 ; P<0.001)$ and subjective knee value $(r=0.66$; $P<0.001)$. Age did not correlate with the clinical outcome or the MRI findings. The lateral trochlear cartilage did, however, deteriorate with longer-term follow-up $(r=0.61$; $P<0.001)$. An unchanged cartilage state was found in 27 knees (follow-up time 45 months). Deterioration was found in 16 knees. Eight knees increased one point (followup time 57 months), five in 2 points (follow-up time 59 months), 3 in 3 points (follow-up time 49 months) and 1 in 4 points (follow-up time 43 months).

The merged dysplasia types $\mathrm{A}$ and $\mathrm{C}$ (without supratrochlear spur) and type B and D (with supratrochlear spur) revealed a slightly better outcome for B and D regarding VAS in sports activity $(P=0.04)$, postoperative Kujala score and subjective knee value $(P=0.02)$. There were no differences regarding instability found between these two groups.

\section{Discussion}

The most important findings of the present study were that patellofemoral stability can be reliably achieved with trochleoplasty, while the behaviour of pain is not predictable. Trochleoplasty is a good treatment option for severe dysplasia. MRI did not prove to be a more accurate tool for predicting the outcome of surgery.

Trochleoplasty is a surgical procedure that aims to correct the trochlear dysplasia in patients suffering from patellofemoral instability. Different studies have reported the success of this intervention [25, 27-29]. The trochlear groove can be well restored as shown in previous studies, and these morphological changes along with the biomechanical benefits can help normalize the patellofemoral articulation $[2,16]$. The few publications in the literature report good results regarding improved patellofemoral stability, although the results are not described in detail. In the study of von Knoch with the largest patients' number and longest follow-up, only one patient with positive apprehension was found, but in their study, the MPFL was sometimes reconstructed [29]. The results of the present study of trochleoplasties without MPFL-reconstruction revealed that most patients achieve stability without redislocations, but in the final clinical examination, a positive apprehension test persisted in almost $25 \%$ of operated knees. With a restored trochlear groove, better tracking of patella can occur during flexion, but as the knee comes close to full extension, the stabilizing action of the MPFL remains absent. Therefore, nowadays, an additional MPFL-reconstruction is performed. The routinely performed VMO reefing is abandoned, because of the missing additional support. Also, cognizant of the problems associated with isolated lateral release, this part of surgery is only performed in very contract situations nowadays [7].

Because of the difficulty in quantifying pain and patient satisfaction with the current scores, which are timeconsuming to acquire and may be biased by the investigator, the "subjective knee value" has been introduced. 
It revealed to be a useful and practicable tool, which is easily obtained, reasonably represents the patient's overall perception of their knee, and correlated very well with the Kujala score in our study.

As the failures after trochleoplasty are attributed to degenerative changes, it was hypothesized that cartilage changes are correlated with the clinical outcome [15, 25, 2729]. In this first detailed report examining the cartilaginous conditions and changes after trochleoplasty using MRI, there was no evidence of postoperative subchondral necrosis or chondrolysis [4, 23]. This confirms the findings of an earlier report examining three arthroscopic cartilage biopsies after trochleoplasty [26]. Although there may be limitations in the interpretation of the postoperative MRI due to artefacts from metallic debris and the Vicryl suture material, the overall survival of the mobilized cartilage is good, at least within the time period analysed here. Changes on the patellar cartilage, particularly in the central area, may result from the change in the loading pattern and the attempts of the patella to adapt the new geometry of the femur. Most interestingly, the followup time had no correlation with the cartilage changes excepting the lateral trochlear cartilage, which is difficult to interpret. It could be the beginning of isolated patellofemoral osteoarthritis, but the length of follow-up in the present study is too short to allow this hypothesis to be confirmed.

Neither the Kujala sore nor MRI changes were found to be predictors of the clinical outcome after trochleoplasty. An interesting finding was the normalization of patella height in the radiographs. The reason that the height of patella normalizes can be found in the fact that some preoperative slightly high-riding patellae now are in the high normal range. Also, some scarification of the Hoffa body can contribute to a lowering of patella.

An unexpected finding was the fact that preoperative pain was not predictive of the postoperative perception of pain. Therefore, in contrast to the conclusion of others, preoperative pain should not be considered a contraindication for trochleoplasty, as there are patients who experience pain relief with this procedure [12]. The analysis of VAS showed that pain symptoms often occur during intense sports activities or heavy labour. Patient performing those activities should at least be informed that the reduction in subjective pain is unpredictable. Previous surgery, often performed in milder types of dysplasia, was not associated with a significantly worse result.

This study shows that severe dysplasia can successfully be treated with trochleoplasty and that MRI is not helpful for a more accurate indication. The only significant predictor of a better outcome after trochleoplasty was the type of trochlear dysplasia. To our knowledge, this is the first time this has been shown in a study. Patients with a trochlear anomaly with a supratrochlear spur (type B and D) showed a significantly better subjective outcome. It may be hypothesized that this is in part due to an unloading effect of the sulcus deepening, resulting in a lower contact pressures in the patellofemoral joint.

Limitations of this study include the small number of the patients with the different types of dysplasia. The indication for trochleoplasty was broad in this series and meanwhile has been narrowed. The fact that type $\mathrm{A}$ and $\mathrm{C}$ dysplasias have been shown to benefit less from trochleoplasty has resulted in a more restricted indication for trochleoplasty, which seems to be more justifiable for type D and severe type B. Currently, a MPFL-reconstruction is performed in addition to the trochleoplasty, and in rare situations with severe patella alta, distalization of the tibial tubercle is considered. Additional medialization of the tubercle seems not to be necessary, as correction of TTTG is realized indirectly through trochleoplasty by the lateralization of its sulcus [16]. Lateral release remains the exception in knees with severe lateral compression and tilting. Patellar instability with milder forms of trochlear dysplasia (type A, C and mild B) are treated with MPFL-reconstruction, with medialization and/ or distalization of the tibial tubercle in the knee of pathological TTTG and/or patella alta.

\section{Conclusion}

In summary, isolated trochleoplasty achieves satisfactory stabilization of the patella, even if $25 \%$ of the knees have clinical findings of residual patella apprehension, probably best treated with additional MPFL-reconstruction. This complex surgery is best indicated for young patients with a severe dysplasia with supratrochlear spur (type D or severe type B) and without highest sports activities or heavy labour. Preoperative pain is not an absolute contraindication and may-even not reliably-be improved with trochleoplasty. MRI analysis revealed no subchondral necrosis but slight progression of chondromalacia on the lateral trochlear facet, but there was no correlation with the clinical outcome of these patients.

Acknowledgments We would like to thank Vaughan Poutawera, $\mathrm{MBChB}$, from the Department of Orthopaedics, Tauranga Hospital, Tauranga, New Zealand, for editing the manuscript. This study was funded internally using departmental funds. There was no external source of funding to bias the assessment of the data.

Conflict of interest The authors declare that they have no conflict of interest.

\section{References}

1. Albee FH (1915) The bone graft edge in the treatment of habitual dislocation of patella. Med Rec 88:257-259 
2. Amis AA, Oguz C, Bull AM, Senavongse W, Dejour D (2008) The effect of trochleoplasty on patellar stability and kinematics: a biomechanical study in vitro. J Bone Joint Surg Br 90:864-869

3. Bereiter HG, Gautier E (1994) Die Trochleaplastik als chirurgische Therapie der rezidivierenden Patellaluxation bei Trochleadysplasie des Femurs. Arthroskopie 7:281-286

4. Biedert RM, Bachmann M (2009) Anterior-posterior trochlear measurements of normal and dysplastic trochlea by axial magnetic resonance imaging. Knee Surg Sports Traumatol Arthrosc $17: 1225-1230$

5. Carrillon Y, Abidi H, Dejour D, Fantino O, Moyen B, Tran-Minh VA (2000) Patellar instability: assessment on MR images by measuring the lateral trochlear inclination-initial experience. Radiology 216:582-585

6. Caton J, Deschamps G, Chambat P, Lerat JL, Dejour H (1982) Patella infera. Apropos of 128 cases. Rev Chir Orthop Reparatrice Appar Mot 68:317-325

7. Christoforakis J, Bull AM, Strachan RK, Shymkiw R, Senavongse W, Amis AA (2006) Effects of lateral retinacular release on the lateral stability of the patella. Knee Surg Sports Traumatol Arthrosc 14:273-277

8. Davies AP, Costa ML, Shepstone L, Glasgow MM, Donell S (2000) The sulcus angle and malalignment of the extensor mechanism of the knee. J Bone Joint Surg Br 82:1162-1166

9. Davies AP, Glasgow MM (2000) Imaging in osteoarthritis: a guide to requesting plain X-rays of the degenerate knee. Knee 7:139-143

10. Dejour D, Le Coultre B (2007) Osteotomies in patello-femoral instabilities. Sports Med Arthrosc 15:39-46

11. Dejour D, Reynaud P, Le Coultre B (1998) Douleurs et instabilité rotulienne. Essai de classification. Méd Hyg 56:1466-1471

12. Dejour D, Saggin P (2010) The sulcus deepening trochleoplastythe Lyon's procedure. Int Orthop 34:311-316

13. Dejour H, Walch G, Neyret P, Adeleine P (1990) Dysplasia of the femoral trochlea. Rev Chir Orthop Reparatrice Appar Mot 76:45-54

14. Dejour H, Walch G, Nove-Josserand L, Guier C (1994) Factors of patellar instability: an anatomic radiographic study. Knee Surg Sports Traumatol Arthrosc 2:19-26

15. Donell ST, Joseph G, Hing CB, Marshall TJ (2006) Modified Dejour trochleoplasty for severe dysplasia: operative technique and early clinical results. Knee 13:266-273

16. Fucentese SF, Schottle PB, Pfirrmann CW, Romero J (2007) CT changes after trochleoplasty for symptomatic trochlear dysplasia. Knee Surg Sports Traumatol Arthrosc 15:168-174
17. Fuchs B, Jost B, Gerber C (2000) Posterior-inferior capsular shift for the treatment of recurrent, voluntary posterior subluxation of the shoulder. J Bone Joint Surg Am 82:16-25

18. Goutallier D, Raou D, Van Driessche S (2002) Retro-trochlear wedge reduction trochleoplasty for the treatment of painful patella syndrome with protruding trochleae. Technical note and early results. Rev Chir Orthop Reparatrice Appar Mot 88: 678-685

19. Kujala UM, Jaakkola LH, Koskinen SK, Taimela S, Hurme M, Nelimarkka O (1993) Scoring of patellofemoral disorders. Arthroscopy 9:159-163

20. Malghem J, Maldague B (1989) Depth insufficiency of the proximal trochlear groove on lateral radiographs of the knee: relation to patellar dislocation. Radiology 170:507-510

21. Masse Y (1978) Trochleoplasty. Restoration of the intercondylar groove in subluxations and dislocations of the patella. Rev Chir Orthop Reparatrice Appar Mot 64:3-17

22. Noyes FR, Stabler CL (1989) A system for grading articular cartilage lesions at arthroscopy. Am J Sports Med 17:505-513

23. Salzmann GM, Weber TS, Spang JT, Imhoff AB, Schottle PB (2010) Comparison of native axial radiographs with axial MR imaging for determination of the trochlear morphology in patients with trochlear dysplasia. Arch Orthop Trauma Surg 130:335-340

24. Schoettle PB, Zanetti M, Seifert B, Pfirrmann CW, Fucentese SF, Romero J (2006) The tibial tuberosity-trochlear groove distance; a comparative study between CT and MRI scanning. Knee 13:26-31

25. Schottle PB, Fucentese SF, Pfirrmann C, Bereiter H, Romero J (2005) Trochleaplasty for patellar instability due to trochlear dysplasia: a minimum 2-year clinical and radiological follow-up of 19 knees. Acta Orthop 76:693-698

26. Schottle PB, Schell H, Duda G, Weiler A (2007) Cartilage viability after trochleoplasty. Knee Surg Sports Traumatol Arthrosc 15:161-167

27. Utting MR, Mulford JS, Eldridge JD (2008) A prospective evaluation of trochleoplasty for the treatment of patellofemoral dislocation and instability. J Bone Joint Surg Br 90:180-185

28. Verdonk R, Jansegers E, Stuyts B (2005) Trochleoplasty in dysplastic knee trochlea. Knee Surg Sports Traumatol Arthrosc 13:529-533

29. von Knoch F, Bohm T, Burgi ML, von Knoch M, Bereiter H (2006) Trochleaplasty for recurrent patellar dislocation in association with trochlear dysplasia. A 4- to 14-year follow-up study. J Bone Joint Surg Br 88:1331-1335 